V.

(Aus dem pharmakologischen Institut zu Wien.)

\title{
Über Phlorhizindiurese und über die Beeinflussung der Phlorhizinzuckerausscheidung durch Diuretica.
}

\author{
Von \\ O. Iroewi und F. Neubauer.
}

(Mit 9 Kurven.)

In einer früheren Mitteilung wurde gezeigt, daß im Gegensatz zu anderen Diuresen die dureh Phlorhizin hervorgerufene die Kochsalzausfuhr nicht steigert, woraus ein besonderer Mechanismus der Phlorhizindiurese ersehlossen wurde Loewi(1). Bei einer Nachprüfnng der Versuche konnte Biberfeld(2) - entgegen der von ihm gewählten Formulierung seiner Ergebnisse - diesen Befund im wesentlichen bestätigen, glaubt aber nach seinen Versuchen annebmen zu müssen, daß die Ursache des Nichtanstiegs der Chloride während der Phlorhizindiurese in einer dem Phlorhizin eignen spezifischen Hemmung der Chloridausfulr liege. Eine derartige Wirkung würde naturgemäß eine Revision der aus der Tatsache des Nichtanstiegs der Chloride gezogenen Sehlußfolgerungen notwendig machen. Deshalb haben wir diese Frage noch einmal einer besonderen Prüfung unterzogen, zumal sich in den früheren Versuchen keinerlei Anhaltspunkte für die Annahme einer derartigen spezifischen Hemmung der NaCl-Ausfuhr ergeben hatten. Bevor wir auf die Versuche selbst eingehen, müssen wir kurz die Unterlagen der Biberfeldschen Seblüsse prüfen.

Zunächst einmal ist in 6 Versuchen $(3,5,8,10,15,16) \mathrm{nach}$ nicht notwendigerweise infolge der Phlorhizinverabfolgung die $\mathrm{NaCl}$ Ausfuhr, mindestens anfänglich, überhaupt nicht gefallen, sondern sogar gestiegen. In 2 weiteren Versuchen $(12,13)$ trat ebensowenig irgendwann ein Abfall ein. Es bleiben demnach nach dem notwendigen Ausschluh des offenbar durch Druckfehler entstellten Versuchs 4 noch 7 Versuche, die von Anfang an eine Verminderung der $\mathrm{NaCl}-\mathrm{Ausfuhr}$ 
anfweisen $(1,2,6,7,9,11,14)$. Ist diese Folge der Phlorhizinwirkung? In 3 davon $(6,9,14)$ waren nach Ausweis der Vorperiode die Chloride schon vor der Phlorhizingabe im Sinken begriffen. In 3 weiteren Versuchen $(1,2,11)$ läßt sich infolge unzureichender Vorperiode ebenfalls nicht entscheiden, ob nicht auch ohne Phlorhizindarreichung die Chloridausscheidung abgenommen hätte. Bleibt also von sämtlichen 16 Versuchen nur ein einziger Versuch (7) als Grundlage für die Annahme einer spezifischen Hemmung der Chloridausfuhr durch Phlorhizin. Und auch diese dürftige Stiitze müssen wir verwerfen; denn die Ergebnisse der Biberfeldschen Versuche verbieten überhaupt Schliusse nach irgend einer Richtung zu ziehen. Nicht nur, daß die Versuche untereinander trotz scheinbar gleicher Anordnung völlig von einander abweichende Ergebnisse lieferten, auch innerhalb des einzelnen Versuches schwanken die Stundenwerte beispiellos und zeigen durchweg nicht den gesetzmäBigen Ablauf, wie es sonst in gutdurchgeführten Diureseversuchen die Regel ist. Biberfeld ist diese Regellosigkeit selbst aufgefallen; er ist geneigt, sie ebenfalls als Ausdrnck einer s p e zifi is ch e n Phlorhizinwirkung anfzufassen. Weit näher liegt doch wohl die Annahme, daß die Regellosigkeit in Besonderheiten der Versuchsanordnung begründet sei, daf etwa die Abgrenzung des Harnes vielleicht infolge der Benutzung männlicher Hunde ${ }^{t}$ ) mißglückt sei. Die Berechtigung, das Unterlaufen von Versuchsfehlern anzunehmen, wird durch eine jüngst erfolgte Mitteilung Biberfelds (3) noch erhöht. Hier verzeichnet er in den Protokollen als höchsten normalen Blutdruckwert von Kaninchen $53 \mathrm{~mm} \mathrm{Hg}$ und Stundendiuresewerte von 11,5 ec bei einem Blutdruck von $26 \mathrm{~mm} \mathrm{Hg}$, ohne daß $\mathrm{hm}$ diese unmöglichen Werte aufgefallen wären.

Darnach ist $u$. E. durch Biberfelds Versuche nichts weniger bewiesen als die Annahme, daß Phlorhizin die Chloridausfuhr spezifisch beeinflusse. Wenn man den Mut hat aus ibnen überhaupt etwas zu sehließen, so kann es nur das sein, daß Phlorbizin ohne jeglichen spezifischen Einflub auf die Chloridausscheidung ist; war diese im Steigen, so steigt sie weiter, war sie im Fallen, so fällt sie weiter, war sie konstant, so bleibt sie es.

\section{Eigene Versuche.}

Die Versuche wurden an weiblichen Hunden ausgeführt, der Harn mittelst Catheter entnommen, die Blase mit 1 proz. Borlösung nachgespült, zur Analyse das Spülwasser mit dem Earn vereinigt. Die Chlorbestimmungen wurden in ublicher Weise nach VolhardSalk ow ski ausgefubrt. Selbstverständlich wurde die Ausscheidung längere Zeit auch vor der (subentanen) Phlorbizininjektion verfolgt,

1) So hat denn auch Loewi ganz im Gegensatz zu der Angabe B.'s nie einen männlichen Hund zu Versuchen, wo catheterisiert werden mute, sondern nur zu Blasenversuchen benutzt, für die naturgemäß das Geschlecht indifferent ist. 
Bulldog $121 / 2 \mathrm{~kg}$ schwer. Erhält am Abend vor dem Versuch I und II je $500 \mathrm{~g}$ Pferdefleisch und $20 \mathrm{~g}$ Fett.

Versuch I (Kontrollversuch). 6. VII. 06.

$\begin{array}{cccc}\text { Zeit } & \text { Harmmenge } & \text { Chloride } \\ & \text { ce } & \text { abs. mg } & 0 / 0 \\ 9-10 & 32 & 89 & 0,28 \\ 10-11 & 32 & 90 & 0,28 \\ 11-12 & 21 & 90 & 0,44 \\ 12-1 & 18 & 94 & 0,52 \\ 1-2 & 12 & 89 & 0,74 \\ 2-4 & 25 & 142 & 0,58 \\ 4-5 & 18 & 56 & 0,31\end{array}$

Versuch II (Phlorhizinversuch). 7. VI, 06.

Zeit Harnmengu

Chloride

Bemerkungen

$\begin{array}{ccccc} & \mathbf{c c} & \text { abs. (mg) } & 0 & \\ 10-11 & 14 & 63 & 0,45 & \\ 11-12 & 13 & 63 & 0,48 & \\ 12-1 & \mathbf{2 1} & \mathbf{6 3} & 0,3 & 12 \mathrm{~h} 1 \mathrm{~g} \text { Phlorh, in } 1 \text { proz. Natr. carb. s. c. } \\ 1-2 & \mathbf{2 3} & \mathbf{6 3} & 0,28 & \\ 2-3 & \mathbf{3 4} & \mathbf{6 0} & 0,18 & \end{array}$
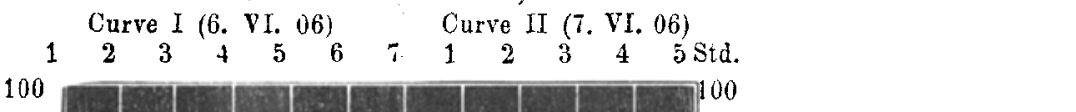

90

80

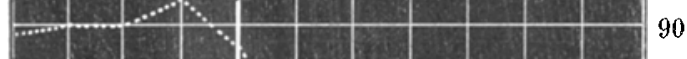

70

60

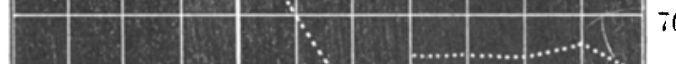

50

40
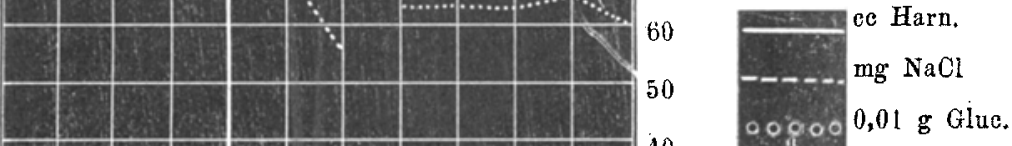

30

20

10

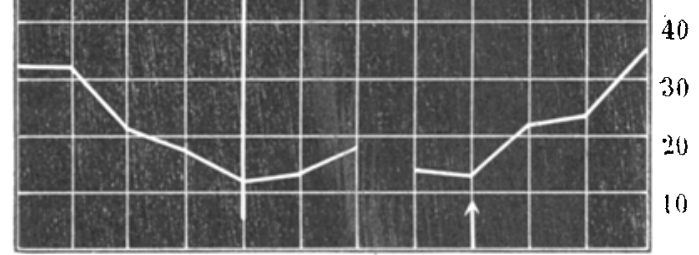

Ig Pblorhizin

subcutan

Der Vergleich der beiden Versuche (und Curven) Tergibt einen völlig gleichen Ablauf der Chloridausscheidung ob Phlorhizin gegeben wurde oder nicht. In II stieg trotz der nach der Phlorhizininjektion einsetzenden Diurese die Chloridausfuhr nicht an, ebensowenig aber sank sie ab. Der Kontrollversuch I demonstriert übrigens auch wie vorsichtig man mit der Beurteilung eines nachträglichen Absinkens der Cbloridausfuhr sein mub.

In den folgenden Versuchen wurde geprüft, ob die Kochsalzausscheidung etwa nach Zufubr größerer Kochsalzmengen durch Pblorhizin eine Hemmung erfahre. 


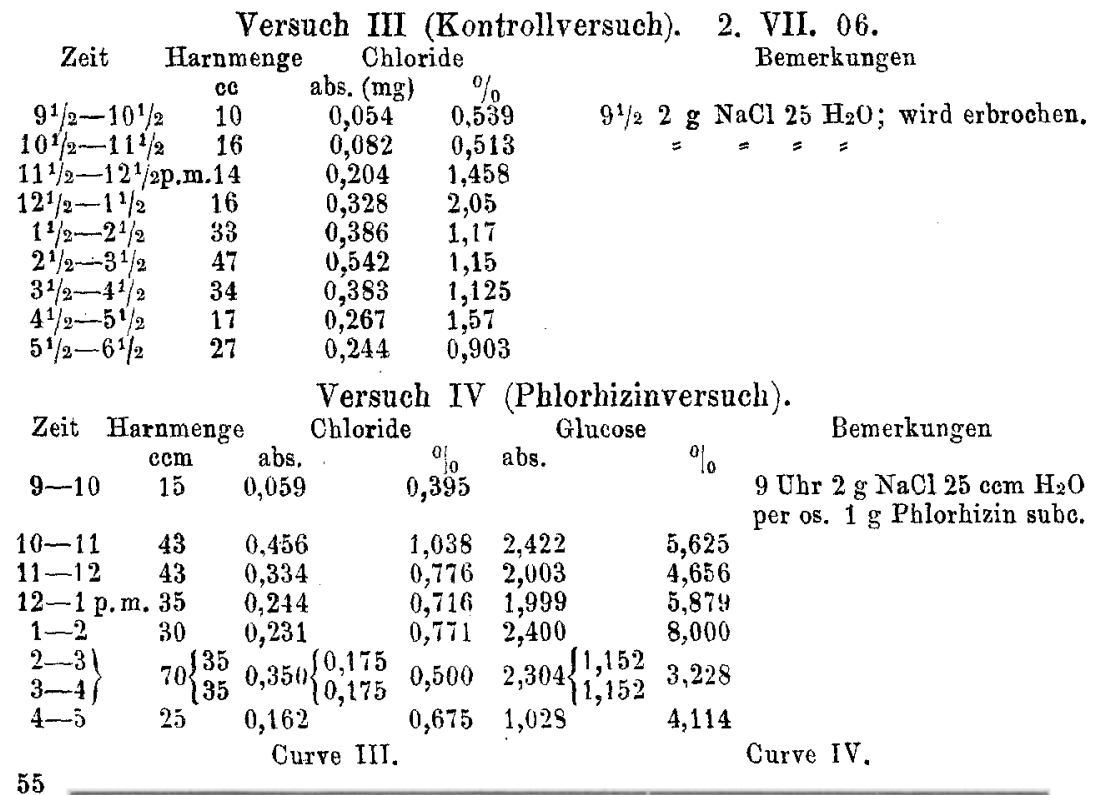

Verlief der Versuch auch insofern nicht ganz rein, als im Kontrollversuch (II) ein Teil des Koebsalzes erbrochen wurde, wofür dann noch einmal $2 \mathrm{~g}$ eingeführt wurden, so zeigt er immerhin eindentig, daß im Phlorhizinversuch (IV) die NaCl-Ausfuhr ebenso 
prompt einsetzt und ebenso abläuft wie im Kontrollversuch. Es wurden in diesem in den sieben der NaCl-Verabfolgung folgenden Stunden 2,35, im Phlorhizinversuch $1,8 \mathrm{~g} \mathrm{NaCl}$ ausgeschieden, sodaß mit Rüeksicht auf die Mehrgabe in Vers. III von einer Hemmung der NaCl-Ausfuhr keine Rede ist.

Versuch $\mathrm{V}$ (Kontrollversuch). 26. VI. 06.

\begin{tabular}{|c|c|c|c|}
\hline Zeit & Iarnmenge & $\mathrm{Cb}$ & ride \\
\hline & $\mathrm{ccm}$ & abs. & $\%$ \\
\hline $10-11$ & 32 & 0,051 & 0,160 \\
\hline $11-12$ & 23 & 0,046 & 0,201 \\
\hline $12-1$ p. $\mathrm{m}$ & 14 & 0,043 & 0,301 \\
\hline $1-2$ & 19 & 0,103 & 0,640 \\
\hline $2-3$ & 27 & 0,333 & 1,12 \\
\hline $3-4$ & 80 & 1,056 & 1,32 \\
\hline $4-5$ & 133 & 3,070 & 2,30 \\
\hline$j-6$ & 164 & 3,788 & 2,31 \\
\hline $6-7$ & 136 & 3,050 & 2,26 \\
\hline
\end{tabular}

Bemerkungen

Versuch VI (Phlorbizinversuch). 27. VI. 06.

\begin{tabular}{|c|c|c|c|c|c|c|c|c|c|}
\hline Zeit & Harnmenge & e Chl & ride & Glu & cose & & & & emerkungen \\
\hline & $\mathrm{ccm}$ & $a b s$ & $\%$ & $a b s$. & $\%$ & & & & \\
\hline $9-10$ & 34 & 0,193 & 0,565 & & & & & & \\
\hline $0-11$ & 14 & 0,077 & 0,553 & & & & & & \\
\hline $1-12$ & 23 & 0,107 & 0,46 & 1,8 & 4,1 & 11 & & $\begin{array}{l}1 \mathrm{~g} \\
1 \mathrm{~g}\end{array}$ & $\begin{array}{l}\text { Phlorhizin s. } \\
\text { NaCl per os. }\end{array}$ \\
\hline $2-1$ & 39 & 0,260 & 0,66 & 2,5 & 6,0 & 12 & $=$ & $2 \mathrm{~g}$ & $=2=$ \\
\hline $1-2$ & 69 & 0,945 & 1,37 & 2,4 & 3,5 & 1 & $=$ & $3 \mathrm{~g}$ & $=$ \\
\hline $2-3$ & 150 & 2,25 & 1,56 & 2,9 & 1,9 & 2 & $=$ & $4 \mathrm{~g}$ & $=$ \\
\hline $3-4$ & 146 & 2,63 & 1,8 & 2,4 & 1,6 & 3 & $=$ & $5 \mathrm{~g}$ & $=$ \\
\hline $4-5$ & 170 & 2,75 & 1,617 & 2,4 & 1,4 & & & & \\
\hline $5-6$ & 115 & 2,162 & 1,88 & 1,9 & 1,6 & & & & \\
\hline
\end{tabular}

Der Vergleich der beiden tadellos verlaufenen Versuche (vergl. Carven $V$ und VI) belehrt darüber, daß die NaCl-Ausscheidung mit und obne Phlorhizin nach allen Richtungen hin identisch ist: in den der ersten $\mathrm{NaCl}$-Darreichung folgenden 7 Stunden wurden ausgeschieden von $15 \mathrm{~g}$

in Versuch III (Kontrollversuch) $=11,47 \mathrm{~g}=77 \%$

$=\quad=I V$ (Phlorhizinversuch) $=11,1 \mathrm{~g}=74 \%$

Im Zusammenbang mit den früher veröffentlichten gentigen die hier mitgeteilten Versuche darzutun, daß die NaCl-Ausscheidung durch Phlorhizineinfuhr in keiner Weise beeinflubt wird: sie verläuft genau, als wenn gar kein Phlorhizin wäre gereicht worden.

Loewi (4) hatte auf Grund seiner Versuche eine Beeinflussung der Phlorhizinzuckerausscheidung durch Diurese geleugnet. Dieser Feststellung widersprach Weber (5) auf Grund seiner allerdings anders angeordneten Versuche. Aus den beiden ersten Versuchen (Tab. VI a s. Curve VII, VIII) dieses Autors läßt sich nicht entnehmen, ob die ohnehin unbeträchtliche Steigerung der Zuckerausfuhr Folge 
Curve V. 1 g Phlorbizin subcutan.

Curve VI.

$1 \mathrm{~g} \quad 2 \mathrm{~g} \quad 3 \mathrm{~g} \quad 4 \mathrm{~g} \quad 5 \mathrm{~g}$ per os

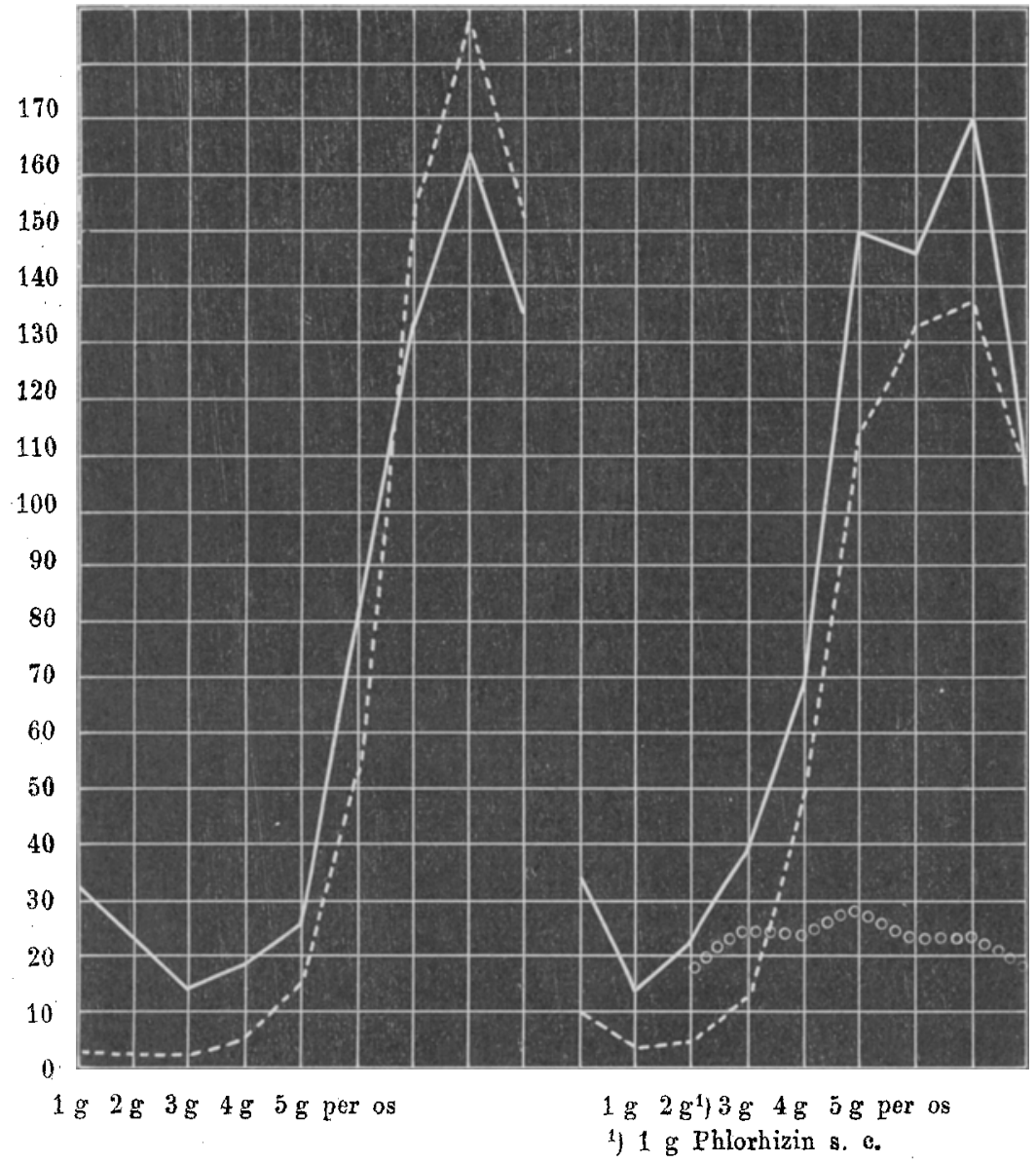

Versuch von Weber. Curve VII. Curve VIII.

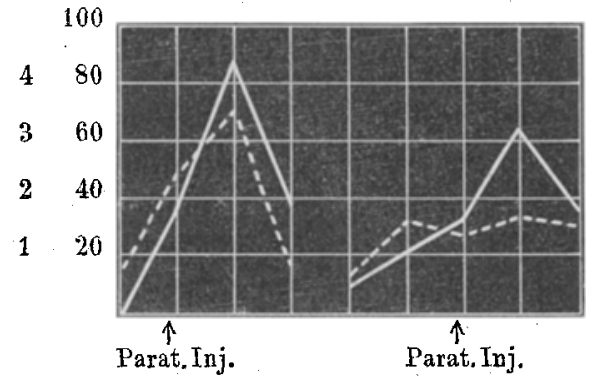

der Diurese war. In Versuch VII war nämlich die Zuckeraiusfuhr bereits ror der Injektion desDiuretioums im Ansteigen begriffen, in Versuch VIII blieb sie durch das Diureticum unbeeinflußt.

In den beiden anderen Versuchen wurde nicht der EinfluB einer aufgesetzten $\begin{array}{lllllllll}15 & 15 & 15 & 15 & 15 & 15 & 15 & 15 & 15 \\ & \text { Min. Diurese geprüft, sondern die }\end{array}$ 
Verschiedenheit der Zuckeransfuhr bei verschieden großer Diurese. In der Tat wurden am 4. III. 1313 ce Harn mit 16,6 g Zueker, am 10. III. 645 ce Harn mit $12,96 \mathrm{~g}$ Zucker ansgeschieden. Diese an sich nicht beträchtliche Differenz beweist aber nichts, da die beiden Beobachtungstage auseinanderlagen und Differenzen um $3 \mathrm{~g}$ Zucker pro die auch bei völlig gleichem Regime die Regel sind.

Den bereits früher von Loewi mitgeteilten schließen wir noch zwei weitere Versuche zur Prüfung dieser Frage an.

Der eine Versuch VI ist bereits oben mitgeteilt worden; aus der Curve ergiebt sich, daB die Zuckerausfuhr im Gegensatz zum $\mathrm{NaCl}$ keineswegs die Schwankungen der Diurese mitmacht.

\section{Versuch VII.}

4. VII. 1906. Hund $121 / 2 \mathrm{~kg}$. 5. VII. letztes Futter u. $1 \mathrm{~g}$ Phlorhizin.

\begin{tabular}{|c|c|c|c|c|c|c|}
\hline \multirow[t]{2}{*}{ Zeit } & \multirow{2}{*}{$\begin{array}{l}\text { Harnmenge } \\
\text { com }\end{array}$} & \multicolumn{2}{|c|}{ Chloride } & \multicolumn{2}{|c|}{ Glucose } & \multirow[t]{2}{*}{ Bemerkungen } \\
\hline & & abs. & $\%$ & abs. & $\%$ & \\
\hline $9-11$ & 39 & 0,203 & 0,521 & 1,029 & 2,63 & \\
\hline $11-1$ & 30 & 0,150 & 0,50 & 1,166 & 3,88 & 1 U̧hr: $30 \mathrm{com} 10 \%$ Natr, nitr. i. \\
\hline $1-3$ & 80 & 0,475 & 0,594 & 1,417 & 1,771 & \\
\hline & 50 & 0,313 & 0,625 & 1,06 & 2,23 & \\
\hline $5-7$ & 41 & 0,225 & 0,549 & 1,08 & 2,63 & \\
\hline
\end{tabular}

Unter dem Einfluß der Natriumnitratinjektion stieg die Diurese auf das doppelte,

Curve IX. $30 \mathrm{ccm} 10 \% \mathrm{Na}-$ mit ibr die Kochsalzansscheidung: die Curve der Zuckerausscheidung blieb unverändert.

Zusammenfassung.

1) Phlorhizinzufubr beeinflu $\beta$ t, im Gegensatz zu anderen diuretisch wirkenden Mitteln, die die Kochsalzausfuhr steigern, diese in keiner Weise; namentlich auch nicht im Sinne einer Hemmung.

2) Die Phlorhizinzuckerausscheidung wird durch anfgesetzte Diurese nicht gesteigert.

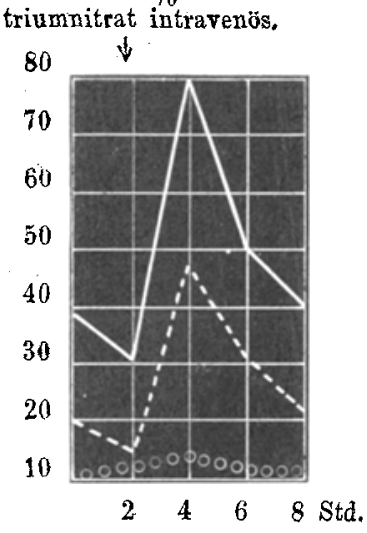

Litteraturverzeichnis.

1) Loewi: ds. Arch. Bd. 50, S. 326, 1903.

2) Biberfeld: Pflügers Arch. Bd. 112, S. 398, 1906.

3) Biberfeld: Pflügers Arch. Bd. 119, S. 341, 1907.

4) Loewi: ds. Arch. Bd. 48, S. 410, 1902.

5) Weber: ds. Arch. Bd. 54, S. 1, 1905. 